\title{
Evolución y letalidad por endocarditis infecciosa en pacientes atendidos en el Hospital Nacional Dos de Mayo
}

\author{
Evelyn Sánchez ${ }^{1}$, César Gutiérrez ${ }^{2}$
}

\section{Resumen}

Palabras clave

\begin{abstract}
Objetivo: Evaluar la evolución y letalidad de los pacientes portadores de endocarditis infecciosa, así como la presencia de complicaciones y la influencia de estas sobre la mortalidad. Diseño: Estudio longitudinal no concurrente. Lugar: Servicio de cardiología del Hospital 2 de Mayo, hospital universitario. Pacientes: Pacientes con diagnóstico de endocarditis infecciosa. Intervenciones: Se revisó las historias clínicas de los pacientes con diagnóstico de endocarditis infecciosa atendidos entre junio de 1999y junio de 2005. Se evaluó los criterios clínicos, de laboratorio yecocardiográficos (criterios de Duke) empleados en el diagnóstico. Para el análisis estadístico, se realizó el análisis descriptivo y comparativo con el empleo de las pruebas de Fisher y tde Student y la comparación de las curvas de Kaplan Meier, para evaluar la supervivencia a través de la prueba de rango logarítmico. Principales medidas de resultados: Evaluación de supervivencia: Complicaciones y mortalidad en pacientes en endocarditis infecciosa. Resultados: Se estudió 75 pacientes, la edad promedio fue 42,1+20,4 años, 66,7\% del sexo masculino. El 93,3\% tenía cardiopatía de base. La presentación clínica incluyó insuficiencia cardiaca congestiva (73,3\%), encefalopatía (17,3\%) y fenómenos embólicos (50,6\%). Se evaluó la evolución de los parámetros de laboratorio, evidenciándose deterioro de la función renal. El ecocardiograma evidenció presencia de vegetaciones en 97,3\% y complicaciones, como rotura valvar (8\%), abscesos valvares (4\%). Durante la evolución, se sometió a cirugía a 14,7\%. La letalidad general fue 14,7\%, la cual estuvo influenciada por el número de complicaciones, en particular las complicaciones sépticas y renales, que impactaron de igual manera en la probabilidad de supervivencia de estos pacientes. Conclusiones: Se registró una letalidad general de 14,7\%. La probabilidad de supervivencia se vio afectada significativamente por el número de complicaciones presentes, entre ellas significativamente las complicaciones sépticas y renales.

Endocarditis; evolución clínica; ecocardiografía.
\end{abstract}

Infectious endocarditis evolution and lethality in patients attended at Dos de Mayo National Hospital

Abstract

Objective: To evaluate infectious endocarditis evolution and lethality as well as complications and their influence on mortality. Design: Non-concurrent longitudinal study. Setting: Dos de Mayo Hospital Cardiology service, a teaching hospital. Patients: Patients

1 Ex residente de cardiología del Hospital Nacional Dos de Mayo. Lima, Perú.

2 Universidad Nacional Mayor de San Marcos, Sección de Epidemiología del Instituto de Medicina Tropical DanielA. Carrión. Lima, Perú. with infectious endocarditis. Interventions: Clinical charts, laboratory and echocardiographic (Dukes criteria) studies of patients with infectious endocarditis attended between June 1999 and June 2005 were reviewed. Fisher and Sudent tests were used for statistical analysis and Kaplan Meier curves comparison and logarithmic range test to evaluate survival. Main outcome measures: Infectious endocarditis complications and mortality. Results: Seventy-five patients were studied, mean age 42,1+20,4 years, 66,7\% males; 93,3\% presented cardiopathy. Clinical presentation included heart failure (73,3\%), encephalopathy (17,3\%), and embolic phenomena (50,6\%). Laboratory evolution showed worsening renal function. Echocardiography demonstrated presence of vegetations in $97,3 \%$ and complications like valve breakage (8\%), valve abscesses (4\%); 14,7\% of patients had surgery. Mortality was $14,7 \%$, caused by renal and septic complications affecting patients' survival. Conclusions: Mortality 
in this group was $14,7 \%$. Survival was affected by the number of complications, mainly renal and septic complications.

Key words: Endocarditis; clinical evolution; echocardiography.

\section{INTRODUCCIÓN}

La endocarditis fue descrita por primera vez en 1885 , por William Osler; las cuatros variables relacionadas con su diagnóstico (cardiopatía predisponente, bacteriemia, fenómeno embólico cutáneo o visceral y proceso endocárdico activo) permanecen como fundamentos hasta hoy. Después de ser denominada bacteriana por varios años, la identificación de otros agentes etiológicos no bacterianos, llevó a la denominación actual de endocarditis infecciosa. Su definición continúa presuponiendo: a) una lesión del endotelio, seguida de depósito de plaquetas y fibrina, dependiente del proceso de coagulación generalmente secundario a flujo anormal, o sea a cardiopatía predisponerte; b) la adhesión de un microorganismo al depósito, esto es el proceso endocárdico activo; y, c) presentación clínica compatible con enfermedad infecciosa, caracterizada por fiebre, escalofríos y mal estado general, con o sin fenómeno embólico ${ }^{(1)}$.

Se presenta preferentemente en pacientes con cardiopatías previas. Las lesiones vegetantes endocárdicas se localizan generalmente en las regiones donde hay menor presión endotelial (cara atrial de las valvas atrio-ventriculares y en la cara ventricular de las sigmoideas). La recidiva en los individuos ya curados refuerza la idea de la existencia de un factor individual de predisposición.

A pesar de ser poco frecuente, con prevalencias anuales estimadas entre 1,6 y $11,6 / 100000$ habitantes $\left({ }^{2,3}\right)$, la endocarditis infecciosa es potencialmente letal, con tasas elevadas entre $15 \%$ y $30 \%$, a pesar del advenimiento de la antibioticoterapia y de los avances de las técnicas operatorias $\left({ }^{4}\right)$.
El diagnóstico de endocarditis infecciosa envuelve un dilema clínico, tanto por la inaccesibilidad de las vegetaciones intracardiacas, cuanto por la ausencia e inespecifidad de las manifestaciones clínicas. Los investigadores del servicio de cardiología de la Universidad de Duke, inspirados en los criterios de Jones de la enfermedad reumática, introdujeron el concepto de criterios mayores y menores $\left({ }^{2}\right)$. Consideraron las vegetaciones demostradas en la ecocardiografía como criterio mayor y sustituirían la ausencia de signos clínicos patognomónicos por una asociación de variables $\left({ }^{5}\right)$. Los criterios de Duke poseen una sensibilidad y especificidad adecuadas ${ }^{(6-8)}$. La inclusión de la ecocardiografía reflejó el cambio de postura frente a la enfermedad, puesto que en el pasado había preocupación en reducir el número de falsos positivos; hoy se busca disminuir los falsos negativos $\left({ }^{9,10}\right)$. El aumento progresivo de la morbilidad y letalidad está relacionado a la demora en el inicio de tratamiento de los casos falsos negativos; y, en los falsos positivos, la utilización de antibióticos dificulta el diagnóstico microbiológico, elevando el costo de tratamiento $\left({ }^{5}\right)$.

El diagnóstico definitivo sigue basándose en el examen anatomopatológico, con la evidencia de la lesión vegetante endocárdica y del agente etiológico, siempre combinado con datos clínicos concomitantes $\left({ }^{11}\right)$.

Por otro lado, la embolia arterial sistémica es la complicación extracardiaca más frecuente en pacientes con endocarditis infecciosa. Es causa importante de morbilidad y mortalidad, y constituye uno de los criterios de intervención quirúrgica. La frecuencia de embolia disminuyó con la introducción del tratamiento antimicrobiano $\left({ }^{12}\right)$.

El presente estudio tuvo por objetivo evaluar la evolución y letalidad de los pacientes portadores de endocarditis infecciosa, así como la presencia de complicaciones y la influencia de estas sobre la mortalidad. 


\section{MATERIALES Y MÉTODOS}

Estudio longitudinal no concurrente. Se seleccionó 75 pacientes del Hospital $\mathrm{Na}-$ cional Dos de Mayo, entre junio de 1999 y junio de 2005, quienes fueron sometidos a examen ecocardiográfico por sospecha de ser portadores de endocarditis infecciosa en fase activa, por criterios clínicos y de laboratorio, y en quienes se formuló el diagnóstico de endocarditis infecciosa. Se escogió solo los pacientes que cumplían con los criterios de Duke (clínico-laboratorialesecocardiográficos).

Se incluyó en el estudio a pacientes con cuadro clínico, pruebas de laboratorio y ecocardiograma compatibles (fiebre, cardiopatía predisponente, soplo de insuficiencia valvar aguda, manifestaciones vasculares, identificación de agente etiológico en los hemocultivos, hallazgos ecocardiográficos, evidencia anatómica o histológica de endocarditis infecciosa) que atendiesen a los criterios de Duke. Pacientes que sufrieran el primer episodio de endocarditis.

Se excluyó los pacientes con cuadro clínico que no atendía a los criterios de Duke, historia clínica con datos incompletos, pacientes en los cuales la ubicación de la endocarditis no fue determinada con precisión, pacientes con episodio repetido de endocarditis.

La indicación del examen ecocardiográfico fue hecha por el médico asistente, de acuerdo a la necesidad clínica. Todos los pacientes fueron sometidos a ecocardiografía convencional transtorácica. Los equipos empleados fueron de la marca Ultramax (Tokio, Japón), modelo 1 000. Se utilizó transductores con frecuencia de 3,5 Mhz.

Se hizo el diagnóstico de endocarditis infecciosa en base al cuadro clínico (fiebre, cardiopatía predisponente, uso ilícito de drogas intravenosas, soplo cardiaco, fenómenos vasculares o inmunológicos), toma de hemocultivo e identificación de com- promiso endocárdico por ecocardiografía transtorácica. Los diagnósticos fueron categorizados de acuerdo a los criterios de Duke.

Se agrupó los antibióticos administrados de acuerdo a cuatro categorías: ceftriaxona + gentamicina, vancomicina + gentamicina, penicilina + gentamicina, otros.

Las complicaciones fueron agrupadas en hemodinámicas y estructurales cardiacas, neurológicas, sépticas, renales, de la terapéutica, del acto quirúrgico; otras complicaciones fueron la embolia pulmonar séptica, insuficiencia de la prótesis, entre otras.

El análisis estadístico tuvo dos fases. La primera fue la fase de estadística descriptiva. La segunda fue la fase de estadística analítica. La asociación entre variables cualitativas se determinó mediante las pruebas de chi cuadrado o prueba exacta de Fisher. Para la comparación de medias, se realizó la prueba Anova para el análisis de los niveles basales con los de evolución de creatinina, hemoglobina, leucocitos y abastonados. Se hizo la comparación de estas variables entre los pacientes que fallecieron y no, mediante la prueba $t$ de Student. Se construyó además curvas de Kaplan Meier y se comparó la función de supervivencia a través de la prueba de rango logarítmico (log rank). En todas las pruebas se consideró como significativo un valor de $p<0,05$. Se usó el paquete estadístico SPSS 11,0.

\section{RESULTADOS}

La edad de los pacientes varió entre 3 y 87 años (promedio de 42,1 20,4 ). El 50\% de los pacientes tenía entre 25 y 55 años. De los 75 pacientes, $50(66,7 \%)$ eran hombres y $25(33,3 \%)$ mujeres.

Se encontró cardiopatía de base en 70 pacientes $(93,3 \%)$. De éstos, la cardiopatía reumática correspondió a $76 \%$ de los 
pacientes, siendo la más frecuente la insuficiencia mitral y aórtica $(22,7 \%)$. Diez pacientes tuvieron defecto congénito asociado, representando $13,3 \%$. La presencia de degeneración mixomatosa manifestada como prolapso de la válvula mitral con diverso grado de insuficiencia mitral se vio en $4 \%$ de los pacientes; el prolapso con insuficiencia mitral severa se observó en $2,7 \%$ y con insuficiencia mitral moderada en $1,3 \%$. El porcentaje de pacientes portadores de prótesis valvares fue $2,7 \%$. La calcificación valvular degenerativa como cardiopatía de base se presentó en $12 \%$, siendo las más frecuentes la calcificación mitral y aórtica.

El tiempo entre el inicio de síntomas y la hospitalización varió entre 6 y 95 días, con una mediana de 21 y media de $24,8 \pm 17,4$. El $50 \%$ de los pacientes inició sus síntomas 5 hasta 30 días antes de su hospitalización. La mayor distribución de tiempo de inicio de síntomas hasta la hospitalización fue de 10 a 20 días ( $24 \%$ de los pacientes).

La manifestación clínica inicial fue insuficiencia cardiaca congestiva (ICC), en $50,7 \%$, fiebre en $45,3 \%$, desorden cerebro vascular (DCV) en $1,3 \%$ y meningoencefalitis en $1,33 \%$. Durante la evolución, la fiebre ocurrió en todos los pacientes; 73,3\% presentó ICC y $17,3 \%$, encefalopatía. Los fenómenos embólicos se presentaron en 38 pacientes, 24 extracerebral, cinco cerebral y nueve pacientes tuvieron embolia cerebral y extracerebral. Los fenómenos embólicos extracerebrales se presentaron con mayor frecuencia a nivel renal, en $44 \%$, seguida de la retina $(21,3 \%)$.

El promedio de creatinina basal fue $0,92 \pm 0,76$; durante el primer control, el promedio fue $1,55 \pm 1,15$ y en el último control, $1,43 \pm 1,43$. La comparación de los resultados mostró una diferencia significativa de la creatinina durante la evolución, evidenciando daño renal $(p<0,01)$.
La hemoglobina promedio al ingreso fue 9,73 , en el segundo control 9,51 y en el último control 9,68; no se encontró diferencia estadísticamente significativa. En cambio, el valor de leucocitos basal promedio fue de 12 674, disminuyendo en el primer control a 10355 ; y, en el último control, llegó a $8891(p<0,01)$. Esto representa una mejoría y normalización de los leucocitos durante la evolución de los pacientes. Esta evolución favorable se evidenció también con la cifra de abastonados, pasando de 5,17 en el primer control a 3,28 en el segundo y finalmente 2,05 $(p<0,01)$.

Tres de los pacientes estudiados (4\%) recibieron antibióticos antes del ingreso al hospital. El agente etiológico no fue identificado en $90,7 \%$, debido a hemocultivos negativos. Los agentes etiológicos identificados fueron estreptococo en cinco pacientes y el estafilococo en dos.

Se observa que el esquema antimicrobiano más frecuentemente empleado fue el de ceftriaxona por seis semanas más gentamicina por dos semanas, seguido de ceftriaxona por cinco semanas más gentamicina por dos semanas. Estos dos esquemas terapéuticos fueron administrados a $54,7 \%$ de los pacientes (Tabla 1).

Por electrocardiografía, 57 pacientes tuvieron ritmo sinusal $(76 \%)$ y 17 fibrilación auricular $(22,8 \%) ; 21 \%$ de los pacientes tuvo hipertrofia y sobrecarga del ventrículo izquierdo; igual porcentaje presentó trastorno de la repolarización del ventrículo izquierdo. Menos frecuentes fueron el crecimiento de aurícula izquierda (8\%) y el bloqueo completo de la rama derecha del haz de Hiss $(5,3 \%)$.

Se muestra las características ecocardiográficas en la Tabla 2. La dimensión de la vegetación en $36 \%$ fue menor de $5 \mathrm{~mm}$, en $32 \%$ fue de $6 \mathrm{~mm}$ a $10 \mathrm{~mm}$ y en $32 \%$ fue mayor de $10 \mathrm{~mm}$. 
Tabla 1. Esquema y tiempo de tratamiento administrado a los pacientes.

\begin{tabular}{|c|c|c|}
\hline Esquema y tiempo de tratamiento & Frecuencia & $\%$ \\
\hline Ceftriaxona 6 ss, gentamicina 2 ss & 21 & 28,0 \\
\hline Ceftriaxona 5 ss, gentamicina 2 ss & 20 & 26,7 \\
\hline Ceftriaxona 4 ss, gentamicina 2 ss & 10 & 13,3 \\
\hline Vancomicina 6 ss, gentamicina 2 ss & 4 & 5,3 \\
\hline Ceftriaxona 4 ss, gentamicina 2 ss & 4 & 5,3 \\
\hline Penicilina 6 ss, gentamicina 2 ss & 2 & 2,7 \\
\hline Penicilina 4 ss, gentamicina 2 ss & 2 & 2,7 \\
\hline Vancomicina 5 ss, gentamicina 2 ss & 2 & 2,7 \\
\hline $\begin{array}{l}\text { Ceftriaxona } 5 \text { ss, vancomicina } 5 \text { ss, } \\
\text { amikacina } 2 \text { ss }\end{array}$ & 1 & 1,3 \\
\hline Ceftriaxona 6 ss, metronidazol 2 ss & 1 & 1,3 \\
\hline Vancomicina 6 ss, clindamicina 2 ss & 1 & 1,3 \\
\hline Ceftazidina 6 ss, vancomicina 6 ss & 1 & 1,3 \\
\hline Vancomicina 2 ss, gentamicina 2 ss & 1 & 1,3 \\
\hline Vancomicina 4 ss, gentamicina 2 ss & 1 & 1,3 \\
\hline Ceftriaxona 3 ss, gentamicina 2 ss & 1 & 1,3 \\
\hline Vancomicina 8 ss, gentamicina 2 ss & 1 & 1,3 \\
\hline $\begin{array}{l}\text { Vancomicina } 8 \text { ss, gentamicina } 2 \text { ss, } \\
\text { clindamicina } 2 \text { ss }\end{array}$ & 1 & 1,3 \\
\hline $\begin{array}{l}\text { Ceftriaxona } 6 \text { ss, gentamicina } 2 \text { ss, } \\
\text { ciprofloxacino } 2 \text { ss }\end{array}$ & 1 & 1,3 \\
\hline Total & 75 & 100,0 \\
\hline
\end{tabular}

ss = semanas

Las complicaciones de la endocarditis infecciosa se presentaron en 70 pacientes $(93,3 \%)$. Una complicación ocurrió en 6 $(8,5 \%)$ pacientes, dos complicaciones en 27 $(38,5 \%)$, tres en $23(32,8 \%)$, cuatro en 11 $(15,7 \%)$, cinco en tres $(4,2 \%)$ pacientes. Predominaron las complicaciones hemodinámicas $(88 \%)$ y estructurales cardiacas $(80 \%)$.

Las principales complicaciones estructurales fueron insuficiencia mitral aguda $(49,3 \%)$, insuficiencia aórtica aguda $(37,3 \%)$, derrame pericárdico $(6,7 \%)$, rotura de cuerda $(6,7 \%)$ y perforación de velo aórtico $(2,7 \%)$. El grado de regurgitación valvular se muestra en la Figura 1.

Las complicaciones neurológicas estuvieron presentes en $18,7 \%$. Fueron principalmente meningitis (cinco pacientes), convulsiones (cuatro pacientes) y DCV (cuatro pacientes).
Tabla 2. Características ecocardiográficas.

\begin{tabular}{lcr}
\hline Características ecocardiográficas & Frecuencia & $\%$ \\
\hline Hallazgos ecocardiográficos & & \\
- Vegetación & 73 & 97,3 \\
- Rotura de cuerda valvar & 6 & 8,0 \\
- Derrame pericárdico & 5 & 6,7 \\
- Absceso perivalvar & 3 & 4,0 \\
- Perforación velo aórtico & 3 & 4,0 \\
- Fístulas entre cámaras & 1 & 1,3 \\
Localización de la endocarditis infecciosa & & \\
- Válvula mitral & 44 & 58,7 \\
- Válvula aórtica & 24 & 32,0 \\
- Válvula tricuspídea & 4 & 5,3 \\
- Endocardio ventricular & 3 & 4,0 \\
- Marcapaso cardiaco & 1 & 1,3 \\
- Prótesis válvula aórtica & 1 & 1,3 \\
- Prótesis válvula mitral & 1 & 1,3 \\
Característica de la verruga & & \\
- Firmemente adherida & & \\
- Prolapsante & 31 & 41,3 \\
- Base fija con borde libre & 23 & 30,7 \\
- Otros & 20 & 26,7 \\
& 1 & 1,3
\end{tabular}

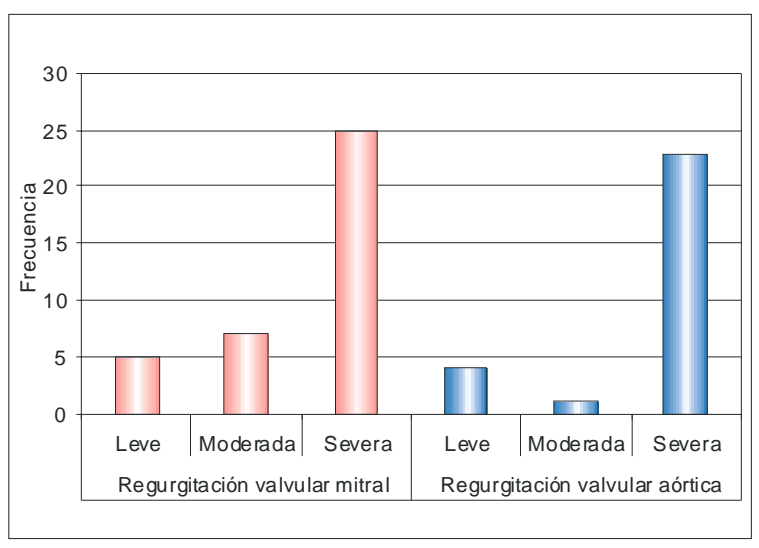

Figura 1. Grado de regurgitación valvular.

Las complicaciones sépticas estuvieron presentes en $18,7 \%$, siendo la principal la psicosis tóxica.

Se realizó 11 intervenciones quirúrgicas. El tiempo entre la hospitalización y la in- 
tervención quirúrgica fue como mínimo 20 días hasta máximo 58 días, con una media de $41,9 \pm 12,3$. El $50 \%$ de los pacientes fue operado entre el día 36 y el día 50. Las principales indicaciones operatorias fueron insuficiencia cardiaca refractaria al tratamiento farmacológico, insuficiencia renal y falla del tratamiento etiológico, con persistencia de hemocultivos positivos.

En el grupo estudiado, la letalidad general fue $14,7 \%$. El tiempo entre el ingreso al hospital y el fallecimiento fue en el menor de los casos 10 días y como máximo 52 días, con una media de $28,7 \pm 13,5$. El $50 \%$ de los pacientes tuvo un tiempo entre el ingreso al hospital y el fallecimiento de 19 a 36 días. La probabilidad de supervivencia de los pacientes, a los 10 días de diagnóstico, fue $98,7 \%$, descendiendo a $91,7 \%$ a los 30 días; a los 70 días, la probabilidad de supervivencia fue $71,5 \%$. La curva de probabilidad de supervivencia de endocarditis infecciosa demostró una disminución progresiva en el tiempo (Figura 2).

Comparando las curvas de Kaplan Meier, la posibilidad de supervivencia es menor en los pacientes que tienen cuatro a seis complicaciones, la cual se ve afectada se-

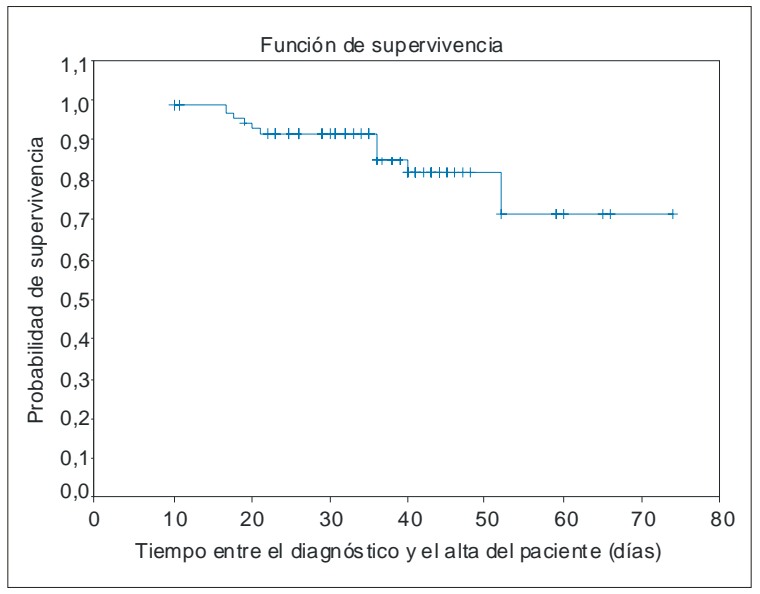

Figura 2. Curva de supervivencia entre el diagnóstico y el alta del paciente. veramente entre los días 10 al 21 (de 92,9\% desciende a 57,1\%), comparado con quienes presentan de una a tres complicaciones $(p<0,001)$ (Figura 3).

Se observó que el mayor número de muertes se dio en el grupo que tenía complicaciones sépticas (53\% de mortalidad), mientras que los que no tenían complicaciones sépticas tuvieron una mortalidad de $5 \%$ (prueba exacta de Fisher $p<0,001$ ). Igualmente, se evidenció una mayor proporción de muertes en el grupo que tuvo complicaciones renales (mortalidad de $70 \%$ ), en comparación con quienes no tenían complicaciones renales (mortalidad de $2 \%$ ) (prueba exacta de Fisher $p<0,001)$.

El valor de creatinina basal en los pacientes fallecidos fue $1,35 \pm 1,93$, mientras que los pacientes que no fallecieron tuvieron una creatinina basal de $0,64 \pm 0,22$. Así, los pacientes que fallecieron tuvieron alterados los exámenes basales de función renal, como predictor de mal pronóstico (t de Student $p=0,046)$.

Con relación a los resultados de los grupos que recibieron antibióticos, los pacientes que usaron la combinación de fármacos

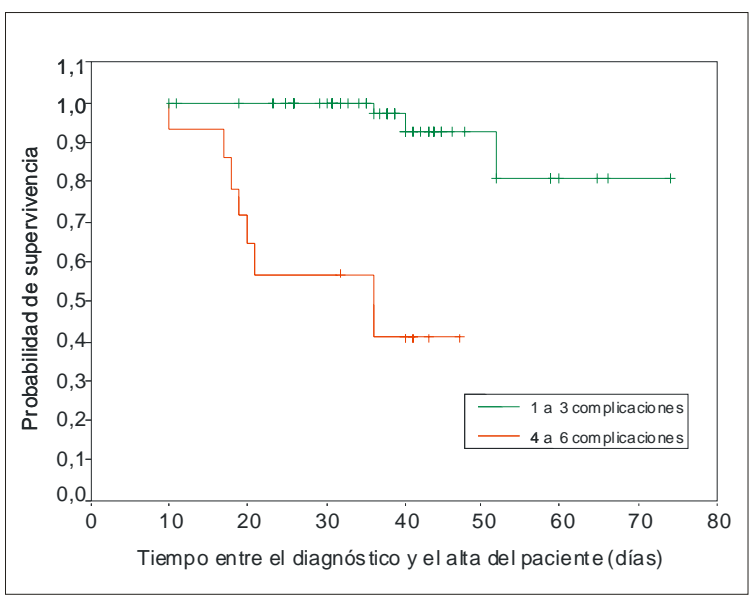

Figura 3. Número de complicaciones y supervivencia (log rank $p<0,001)$. 


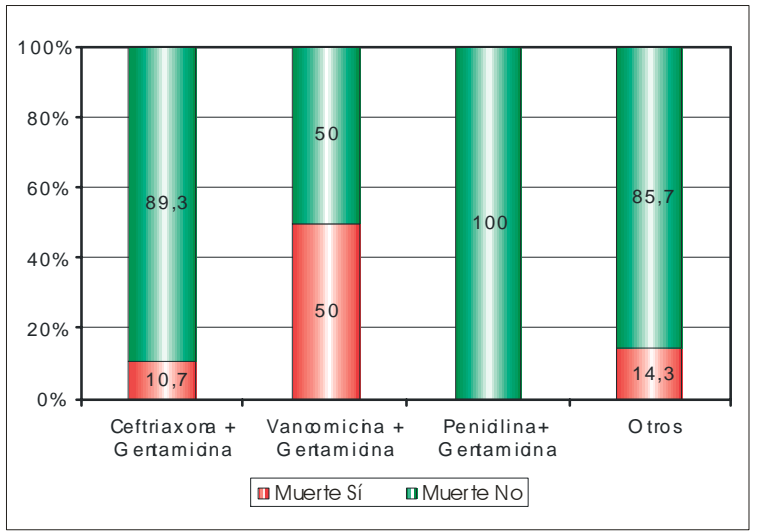

Figura 4. Relación entre la combinación de fármacos antimicrobianos y la letalidad.

vancomicina + gentamicina tuvieron una mayor proporción de fallecimientos $(50 \%)$, al compararlos con los otros grupos, como ceftriaxona + gentamicina $(10,7 \%)$ u otros, $14,7 \%$ (chi cuadrado $p=0,025$ ) (Figura 4).

\section{DISCUSIÓN}

El grupo de pacientes con el diagnóstico de endocarditis infecciosa presentó una media de edad de $42,1 \pm 20,4$, comparable con las casuísticas de otros estudios revisados. El sexo masculino predominó en relación 2:1, semejante a las observaciones de otros autores $\left({ }^{9,13}\right)$. Hubo predominio de la válvula nativa como antecedente cardíaco previo, específicamente en la válvula nativa con lesión reumática (76\%); entre las otras anormalidades presentes se encontró las calcificaciones valvulares en $14 \%$, defectos congénitos en $13 \%$ y prótesis valvulares en $2,7 \%$. Este hallazgo es importante, debido a que otras series muestran mayor número de pacientes portadores de prótesis $\left({ }^{14}\right)$.

La letalidad general de $14,7 \%$ es relativamente baja, teniendo en cuenta que estos pacientes han sobrevivido con tratamiento médico y a muchos de ellos no se les realizó cirugía, a pesar de tener indicación, hasta el término del estudio.

En nuestros pacientes, el tiempo transcurrido entre el inicio de los síntomas hasta su hospitalización fue de 6 a 95 días, con una media de 24,8 , similar a las medias de 25 a 33 días citadas en la literatura $\left({ }^{15}\right)$. Un estudio reveló que el menor intervalo de tiempo entre el inicio de los síntomas y la introducción de los antibióticos ocurrió en los pacientes con evolución fatal, en comparación a los pacientes sobrevivientes $\left({ }^{15}\right)$. Otro estudio mostró que los factores que contribuyen para un mayor periodo entre el inicio de los síntomas y la hospitalización en algunos pacientes, incluye la baja gravedad de los síntomas, una forma más benigna de la enfermedad, la ausencia de complicaciones, el retardo del paciente en buscar la atención médica $y$, también, la dificultad diagnóstica de la enfermedad en su forma más rara. Mostró también que, el periodo entre el inicio de los síntomas y la hospitalización fue menor cuando la endocarditis ocurrió en válvula protésica, fue causada por estafilococo áureo o cuando fue fatal $\left({ }^{16}\right)$. Este hallazgo sugiere que, por la mayor gravedad de los pacientes, los que fueron tratados más precozmente eran portadores de endocarditis aguda y presentaban un riesgo mayor de complicaciones y muerte.

Las prótesis valvares ofrecen mayores dificultades para el diagnóstico, no solo por la ocurrencia frecuente de artefactos, sino también porque son materiales de mayor densidad acústica. Otra dificultad es el menor tamaño de la vegetación en las prótesis. El examen ecocardiográfico de la prótesis requiere una adecuada capacidad de resolución del equipo y pericia del operador. En el estudio, solo hubo dos pacientes portadores de prótesis, uno con prótesis aórtica y otro con prótesis mitral y aórtica. Otra limitación, poco referida pero importante, es la combinación de dos o más pró- 
tesis en un mismo paciente, principalmente en prótesis mitral y aórtica. La sombra acústica causada por la primera sobre la segunda impone dificultades, sobre todo para el análisis de la válvula aórtica.

La mayoría de los pacientes tenía lesiones previas $(93,3 \%)$. La lesión previa predominante fue la cardiopatía reumática, lo que no concuerda con otras publicaciones. Una lesión de baja frecuencia fue la degeneración mixomatosa de la válvula, presente solo en $4 \%$, a diferencia de los estudios extranjeros, en donde esta tiene mayor prevalencia. La alta frecuencia de enfermedad reumática es lo esperado en nuestro medio.

Observamos que la función renal, evaluada por la creatinina, durante la evolución fue deteriorándose con respecto al valor basal, con un $p<0,05$. Con respecto al valor de los leucocitos, estos tuvieron una variación hacia valores normales durante la evolución, en la mayoría de pacientes, con un $p=0,001$. Del mismo modo se comportaron los abastonados, que evolucionaron a valores normales en la mayoría de pacientes, con un $p=0,001$.

La frecuencia de fallecidos fue mayor en los pacientes que presentaban cuatro a seis complicaciones con respecto a los que presentaban una a tres complicaciones, lo cual fue significativo, con $p<0,001$. Del mismo modo, se observó una mayor frecuencia de muerte en los pacientes que presentaban complicaciones sépticas y complicaciones renales, $p<0,001$. En nuestro datos se observa también que, los pacientes con creatinina basal mayor de lo normal presentaron mayor frecuencia de muerte $(p=$ $0,046)$. La probabilidad de supervivencia de endocarditis se vio afectada por el número de complicaciones presentes, la existencia de complicaciones sépticas y renales, lo cual fue significativo, con un valor $p<0,001$.

Dentro de las limitaciones del presente estudio podemos mencionar que, los pacien- tes representan a un grupo en que han predominado portadores de válvula nativa, específicamente válvula nativa con lesión reumática y diagnóstico de endocarditis infecciosa, que no necesariamente pueden ser representativos de otras circunstancias clínicas que correspondan con el diagnóstico de endocarditis infecciosa. El riesgo potencial de datos incompletos de seguimiento o de complicaciones no fatales está presente. Pese a contar con una casuística adecuada para algunos eventos, es posible que no haya alcanzado el número para tener un mayor poder estadístico, por lo que algunas asociaciones no han podido ser demostradas.

En conclusión, los pacientes evolucionaron con una letalidad general de $14,7 \%$. Se observó una mayor cantidad de fallecidos en el grupo de pacientes que emplearon antibióticos de mayor espectro, como vancomicina + gentamicina. La probabilidad de supervivencia se vio afectada significativamente por la presencia del número de complicaciones presentes, entre ellas destacaron de manera significativa las complicaciones sépticas y renales.

\section{REFERENCIAS BIBLIOGRÁFICAS}

1. Aranki SF, Adams DH, Rizzo RJ, Couper GS, Kinchla NM, Gildea JS, et al. Determinants of early mortality and late survival in mitral valve endocarditis. Circulation. 1995;92(suppl II):143-149.

2. Durack DT, Lukes AS, Bright DK. New criteria for diagnosis of infective endocarditis: utilization of specific echocardiographic findings. Duke Endocarditis Service. Am J Med. 1994;96:200-209.

3. Fenollar F, Gauduchon V, Casalta JP, Lepidi H, Vandenesch F, Raoult D. Mycoplasma endocarditis: two case reports and a review. Clin Infect Dis. 2004;38:e21-4.

4. Lengyel M. The impact of transesophageal echocardiography on the management of aortic root abscess. Br Heart J. 1997;72:204-211.

5. Bosshard PP, Kronenberg A, Zbinden R, Ruef C, Böttger EC, Altwegg M. Etiologic diagnosis of infective endocarditis by broad-range polymerase chain reaction: a 3-year experience. Clin Infect Dis. 2003;37:167-172.

6. Ccchine I, Chinaglia A, Pamari I, Brusasco G, Bobbio M, Teo R, et al. New diagnostic criteria for infective endocarditis. 
A study of sensitivity and specifity. Eur Heart J. 1997; 18:1149-1156.

7. Hoen B, Selton-Suty C, Lacassin F, Etienne J, Briacon S, Leport C, et al. Infective endocarditis in patients with negative blood cultures: analysis of 88 cases from a one-year nation wide survey in France. Clin Infect Dis. 1995;20:501-506.

8. Ionescu AA, Butchart EG. The vegetation that was not vegetation: excusable misinterpretataion of transesphageal echocardiography finding in late infective endocarditis of a prosthetic mitral valve. J Am Soc Echocardiogr. 2000;13:322-324.

9. Delahaye JP, Ecochard R, Gevigney G, Barjhoux C, Malquarti $\mathrm{V}$, Saradarian $\mathrm{W}$, et al. The long term prognosis of infective endocarditis. Eur Heart J. 1995;16(suppl B):48-53.

10. Di Salvo G, Habbib G, Pergola V, Avierinos JF, Philip E, Casalta JP, et al. Echocardiography predicts embolic events in infective endocarditis. J Am Coll Cardiol. 2001;37:10691076.

11. Gillinov AM, Shah RV, Curtis WE, Stuart RS, Cameron DE, Baumgartner WA, et al. Valve replacement in patient with endocarditis and acute neurologic deficit. Ann Thorax Surg. 1996;61:1125-1130.

12. Horstkotte D, Follath F, Gutschik E, Lengyel M, Oto A, Pavie A, et al. Guidelines on prevention, diagnosis and treatment of infective endocarditis. European Heart Journal. 2004;25:267-276.
13. Dodds GA, Sexton DJ, Durack DT, Bashore TM, Corey GR, Kisslo J. Negative predictive value of the Duke criteria for infective endocarditis. Am J Card. 1996;77:403-407.

14. Gauduchon V, Chalabreysse L, Etienne J, Celard M, Benito $\mathrm{Y}$, Lepidi $\mathrm{H}$, et al. Molecular diagnosis of infective endocarditis by PCR amplification and direct sequencing of DNA from valve tissue. J Clin Microbiol. 2003;41:763766.

15. Kjerulf A, Tvede M, Aldershvile J, Hoiby N. Bacterial endocarditis at a tertiary hospital-how do we improve diagnosis and delay of treatment? A retrospective study of 140 patients. Cardiology. 1998;89:79-86.

16. Issa VS, Fabri Jr J, Pomerantzef PMA, Grinberg M, PereiraBarreto AC, Mansur AJ. Duration of symptoms in patients with infective endocarditis. Int J Cardiol. 2003;110:334339.

Manuscrito recibido el 23 de mayo de 2006 y aceptado para publicación el 20 junio de 2006.

Correspondencia:

Dra. Evelyn Sánchez Hurtado

Las Pecanas 129, La Molina

Lima 12, Perú

Correo-e:evelynsanchez@yahoo.com 